\title{
A redox probe screens MTHFD1 as a determinant of gemcitabine chemoresistance in cholangiocarcinoma
}

\author{
Ruogu Pan', Zhiqing Yuan', Yingbin Liu', Xuxu Sun², Guiyang Wang ${ }^{1}$, Xiaopen Wang ${ }^{1}$, Junwen Qu', Jian Wang ${ }^{1}$,
}

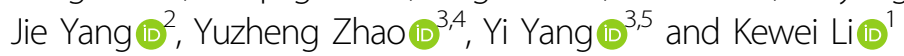

\begin{abstract}
Cholangiocarcinoma (CCA) is a type of solid tumor derived from the bile duct epithelium that features universal gemcitabine resistance. Here, we utilized a gene-encoded ROS biosensor probe (HyPer3 probe) to sort subpopulations with different redox statuses from CCA cells. The isolated HyPer-low subpopulation CCA cells, which exhibited relatively lower cellular ROS levels, exhibited higher chemoresistance to gemcitabine than HyPer-high subpopulation CCA cells in vitro and in vivo. Mechanistically, increased expression of MTHFD1 was found in HyPer-low cells. Knocking down MTHFD1 in HyPer-low cells enhanced cellular ROS and restored sensitivity to gemcitabine. Furthermore, the MTHFD1 inhibitor antifolate compound methotrexate (MTX) increased cellular ROS, and combining gemcitabine with MTX effectively suppressed cholangiocarcinoma cell growth. In summary, the MTHFD1 level mediated the heterogeneous cellular redox status in CCA, which resulted in chemoresistance to gemcitabine. Our data suggest a novel strategy for CCA chemotherapy.
\end{abstract}

\section{Introduction}

Cholangiocarcinoma (CCA), a molecularly heterogeneous neoplasm that arises from the epithelial cells of the bile duct, is one of the most prevalent digestive system neoplasms in adults with high mortality and poor prognosis ${ }^{1,2}$. The incidence and mortality rates of CCA have increased globally, especially in northeastern Asia. Data from the National Cancer Institute show that the 5-year survival rate of CCA is $\sim 8-10 \%^{3}$. Most patients are at the advanced or metastatic stage and have already lost

\footnotetext{
Correspondence: Jie Yang (yangjieyj@shsmu.edu.cn) or

Kewei Li (keweipig@126.com)

'Department of Biliary and Pancreatic Surgery, Renji Hospital, School of Medicine, Shanghai Jiaotong University, Shanghai 200127, China

${ }^{2}$ Department of Biochemistry and Molecular Cell Biology, State Key Laboratory of Oncogenes and Related Genes, Shanghai Key Laboratory forTumor

Microenvironment and Inflammation, Shanghai Jiaotong University School of Medicine, Shanghai 200025, China

Full list of author information is available at the end of the article

These authors contributed equally: Ruogu Pan, Zhiqing Yuan

Edited by I. Amelio
}

surgical indications ${ }^{4}$. For these patients, chemotherapy is the major nonsurgical approach ${ }^{5,6}$.

Gemcitabine was used to treat various solid malignant tumors, including CCA, due to its nucleoside analog deoxycytidine (2',2'-difluoro 2'-deoxycytidine) property ${ }^{7}$. The regimen that combines gemcitabine with cisplatin or other reagents is the first-line treatment for patients with locally advanced and metastatic bile duct tumors, but poor sensitivity to gemcitabine is commonly observed among them ${ }^{5,6}$. Along with an increasing number of signaling pathways and several targets, such as PI3K/Akt, Erk, and NF- $\mathrm{kB}$, identified in gemcitabine resistance in CCA cells, the determining mechanism needs to be uncovered $^{8}$.

Notably, it has been reported that reactive oxygen species (ROS) can confer sensitivity to gemcitabine to pancreatic cancer cells and gallbladder cancer cells ${ }^{9,10}$, and it is well known that the cellular redox status is correlated with the chemoresistance of various solid tumors, such as $\mathrm{HCC}^{11}$, lung cancer ${ }^{12}$, esophageal cancer, stomach cancer,

\section{(c) The Author(s) 2021}

(c) (i) Open Access This article is licensed under a Creative Commons Attribution 4.0 International License, which permits use, sharing, adaptation, distribution and reproduction cc) in any medium or format, as long as you give appropriate credit to the original author(s) and the source, provide a link to the Creative Commons license, and indicate if changes were made. The images or other third party material in this article are included in the article's Creative Commons license, unless indicated otherwise in a credit line to the material. If material is not included in the article's Creative Commons license and your intended use is not permitted by statutory regulation or exceeds the permitted use, you will need to obtain permission directly from the copyright holder. To view a copy of this license, visit http://creativecommons.org/licenses/by/4.0/. 
and cervical cancer ${ }^{13-15}$. However, the relationship between ROS and CCA chemoresistance is still unclear.

The cellular redox status maintaining dynamic equilibrium under the physical state results from ROS production and elimination systems ${ }^{16}$. The generation system simply consists of two categories: biological processes such as mitochondrial oxidative metabolism and signal transduction processes such as a cellular response to cytokines and xenobiotics ${ }^{17}$. Compared to the simple ROS generation system, the ROS elimination system operates in an inverse way and consists of two main ways ${ }^{17}$. One of these is to directly eliminate ROS and includes superoxide dismutases (SOD), glutathione peroxidase (GPx), glutathione S-transferase pi (GST-pi), metallothionein-3 (MT3), ferritin heavy chain (FHC), and dihydrodiol dehydrogenase (DDH1 or AKR $1 C 1)^{18,19}$. Meanwhile, the generation of NADP/ $\mathrm{NADPH}$, such as the pentose phosphate pathway and methylenetetrahydrofolate dehydrogenase (MTHFD1), also contributes to the balance of the cellular redox status $^{20,21}$. The long-term unsolved questions are what is the heterogeneous cellular redox status in various cancer cells and how do intrinsic ROS affect the constitutive chemoresistance.

To uncover the correlation between the intrinsic cellular redox status and CCA gemcitabine chemoresistance, we applied a novel tool, the HyPer3 probe, to sort out cellular redox status differences in a subpopulation of CCA cells and found that HyPer-low CCA cells exhibited higher gemcitabine chemoresistance than others with higher MTHFD1 expression. Since MTHFD1 is associated with NADPH production and the enzyme providing onecarbon unit derivatives of tetrahydrofolate, the key role and mechanism of the cellular redox status and MTHFD1 in CCA cell chemoresistance to gemcitabine is worth noting.

\section{Materials and methods}

\section{Cell culture and reagents}

The cholangiocarcinoma cell line QBC939 and HUCCT1 were purchased from the Cell Bank of the Chinese Academy of Sciences (Shanghai, China). The human embryonic kidney 293T (HEK293T) cells were purchased from the American Type Culture Collection. QBC939 and HUCCT1 were all MTHFD1 WT genotype $\left(\mathrm{MTHFD}^{+/+}\right)$. QBC939, HUCCT1, and HEK293T cells were cultured in Dulbecco's modified Eagle's medium (Gibco). All cell lines were supplemented with $10 \%$ fetal bovine serum (Gibco), penicillin $(100 \mathrm{mg} / \mathrm{ml})$ and streptomycin $(100 \mathrm{mg} / \mathrm{ml})$ and were incubated in a humidified chamber with $5 \% \mathrm{CO}_{2}$ at $37^{\circ} \mathrm{C}$. Gemcitabine, Methotraxate, and puromycin were purchased from MedChemExpress (MedChemExpress, Monmouth Junction, NJ).

\section{Plasmids and siRNA and}

The ORF sequences of HyPer3 were cloned into the $\mathrm{pCDH}$ vector with puromycin in the C-terminus. MTHFD1-targeting siRNA and non-specific control siRNA (siNC) used in this study were obtained from Biochemistry and Molecular Cell Biology, Shanghai Jiaotong University School of Medicine.

\section{Cell apoptosis assays}

QBC939 and HUCCT1 cells were plated in dishes or microplates, overnight and treated with gemcitabine or vehicle for $48 \mathrm{~h}$ under 1 and $10 \mu \mathrm{M}$. After that, all cells were collected by trypsinization without EDTA, and $2 \times$ $10^{6}$ cells were doubly stained with annexin-V-APC/PI (BD Bioscience) and analyzed by fluorescence-activated cell sorting analysis.

\section{Experimental animal model}

For subcutaneous injection models, cells were resuspended in $50 \mu \mathrm{L}$ phosphate-buffered (PBS) and mixed with $50 \mu \mathrm{L}$ Matrigel (BD Biosciences, Billerica, MA), then the cell mixture was implanted subcutaneously into the both flank of the 6-week-old mice (male BALB/c nude mice). Tumors were measured every two days and calculated by the following formula: Volume $=0.5 \times$ Width $^{2} \times$ Length, with four mice per group. The tumor volume and weight presented as the means \pm S.D $(n=3-4)$. All procedures involving animals were approved and performed in accordance with the Animal Care and Use Committee of Shanghai Jiaotong University.

\section{Chemoresistance assay}

Chemotherapy-induced cytotoxicity was assayed by the Cell-Counting Kit-8 (MedChemExpress, Monmouth Junction, NJ). Briefly, cells were plated in 96-well plates at $5 \times 10^{3}$ cells/well to attach overnight, then treated with Gemcitabine (Gem; MedChemExpress, Monmouth Junction, NJ) at various concentrations. The cell-counting kit-8 assay was performed according to protocols from manufacturer. All experiments were performed independently at least 3 times, and each experiments contained triplicates.

For in vivo chemoresistance nude mice model, $5 \times 10^{6}$ QBC939 HyPer-low cells or $5 \times 10^{6}$ QBC939 HyPer-high cells were resuspended in $50 \mu \mathrm{L}$ PBS and mixed with $50 \mu \mathrm{L}$ Matrigel, then implanted subcutaneously into the left flank of 6-week-old mice (male BALB/c nude mice). After 1 week, the mice were intraperitoneally injected with PBS or gemcitabine $(50 \mathrm{mg} / \mathrm{Kg}$ body weight) twice per week for two weeks. Tumor growth was measured every two days and calculated by the following formulation: Volume $=0.5 \times \mathrm{Width}^{2} \times$ Length, with four mice per group. The tumor volume and weight presented as the means \pm S.D $(n=3-4)$. 
For in vivo anti-chemoresistance nude mice model, $5 \times$ $10^{6}$ QBC939 HyPer-low cells or $5 \times 10^{6}$ QBC939 HyPerhigh cells were resuspended in $50 \mu \mathrm{L}$ PBS and mixed with $50 \mu \mathrm{L}$ Matrigel, then implanted subcutaneously into the left flank of 6-week-old mice (male BALB/c nude mice). After 1 week, the mice were intraperitoneally injected with gemcitabine $(50 \mathrm{mg} / \mathrm{Kg}$ body weight) twice per week for two weeks. Tumor growth was measured every two days and calculated by the following formulation: Volume $=0.5 \times \mathrm{Width}^{2} \times$ Length, with four mice per group. The tumor volume and weight presented as the means \pm S.D $(n=3-4)$.

\section{Flow cytometric analysis}

The cell were transfected with HyPer3 probe plasmid $48 \mathrm{~h}$ before. The cells were treated with $\mathrm{H}_{2} \mathrm{O}_{2}$ or DTT and analyzed using a flow cytometer (BD, Biosciences). The cells treated with PBS was served as a control. For cell sorting, its based on the ratio of F488nm/F405nm, followed by sorting with a flow cytometer (BD, Biosciences).

\section{Immunofluorescence staining}

Tissues were seeded on the cover slides and fixed in $4 \%$ paraformaldehyde over night and treated with $0.05 \%$ Triton X-100. Then the tissue were blocked for $30 \mathrm{~min}$ in $10 \%$ bovine serum albumin (BSA; Sigma-Aldrich) in PBS and then incubated with fluorescence-conjugated secondary antibodies at room temperature for $1 \mathrm{~h}$. DAPI (4,6-diamidino-2-phenylindole) was used to stain nuclei. Immunofluorescence signals were captured by a fluorescence microscope (Leica Biosystems, Nubloch, Germany).

\section{Western-blot assays}

Immunoblotting was performed using standard procedures. Cell lysates were prepared in radioimmunoprecipitation lysis buffer (50 mM Tris, $\mathrm{pH} 7.4$, $150 \mathrm{mM} \mathrm{NaCl}, 1 \% \mathrm{NP}-40,0.1 \%$ sodium dodecyl sulfate (SDS), $2 \mu \mathrm{M}$ EDTA) containing proteinase inhibitor and were quantified with the Micro BCA Protein Assay Kit (Thermo Fisher Scientific). Aliquots of $20 \mu \mathrm{g}$ protein were electrophoresed through $10 \%$ or $15 \%$ SDS polyacrylamide gels and were then transferred to polyvinyl difluoride membranes (Millipore), followed by blocking in 5\% skim milk at room temperature for $1 \mathrm{~h}$ and incubated with primary antibodies at $4{ }^{\circ} \mathrm{C}$ overnight. Secondary antibodies were labeled with horseradish peroxidase, and the signals were detected using the ECL Kit (Millipore). The images were analyzed using Image 1.43 software. $\beta$-Actin served as an internal control for the whole-cell lysates. Antibody against MTHFD1 (A8661, dilution 1:1000) was from Abclonal; $\beta$-Actin (A1978, dilution 1:1000) was from Sigma-Aldich.
RNA extraction and real-time quantitative PCR (RT-qPCR)

Total RNA was extracted from cells using Trizol Reagent (Life technology) following the manufacturer's protocol, and $1 \mu \mathrm{g}$ of total RNA was reverse transcribed using the PrimerScript RT Reagent Kit (TaKaRa) into cDNA. RT-qPCR was performed in Roche Light Cycler 480 Real-Time PCR system (Roche). The Ct values obtained from different samples were compared using $2^{-\triangle \triangle \mathrm{Ct}}$ method, and $\beta$-Actin or GAPDH served as an internal reference gene.

\section{NADPH/NADP ${ }^{+}$assay}

Cellular ratio of $\mathrm{NADPH} / \mathrm{NADP}^{+}$was assayed by the NADP/NADPH Assay Kit (Abcam). Briefly, sorted cells were plated in 96 -well plates at $5 \times 10^{3}$ cells/well to attach overnight. The NADP/NADPH Assay Kit was performed according to protocols from manufacturer. All experiments were performed independently at least 3 times, and each experiment contained triplicates.

\section{Statistical analysis}

Data were presented at the mean \pm S.D. one-sample Kolmogorov-Smirnov test was applied for normally distributed data examination. For normal distribution data, two tailed-student's $t$ test was applied to compare the difference between two groups, and one-way analysis of variance test was applied to compare the difference among three or more groups; for non-parametric data, Mann-Whitney $U$ test (data with abnormal distributions) was applied. For survival analysis, the Kaplan-Meier method and log-rank test were applied to determine the OS. Fisher's exact tests were applied to analyze the correlation. All statistical calculation was performed using SPSS software package (version 23.0, IBM SPSS), and a $P<0.05$ was considered to be statistically significant.

\section{Results}

The redox status was heterogeneous in cholangiocarcinoma, which was revealed by HyPer3

The HyPer3 probe is a genetically encoded biosensor that enables real-time imaging of hydrogen peroxide with whole or individual compartments of the $\mathrm{cell}^{22-24}$. The probe consists of two OxyR domains with one integrated cPYFP domain. Once hydrogen peroxide reacts with the OxyR domain in the C-terminus, the disulfide bond between Cys199 and Cys208 immediately forms ${ }^{22,25}$ (Fig. 1A). Following disulfide bond formation by hydrogen peroxide, the integrated cPYFP domain undergoes conformational changes and is finally transmitted to the fluorescent protein $^{22}$. Furthermore, the HyPer3 probe also acts as a ratiometric sensor that can vividly reflect the above intramolecular reorganizations. The spectral characteristics of the HyPer3 probe possess two fluorescence excitation peaks (F488 $\mathrm{nm}$ and F405 nm) and one 


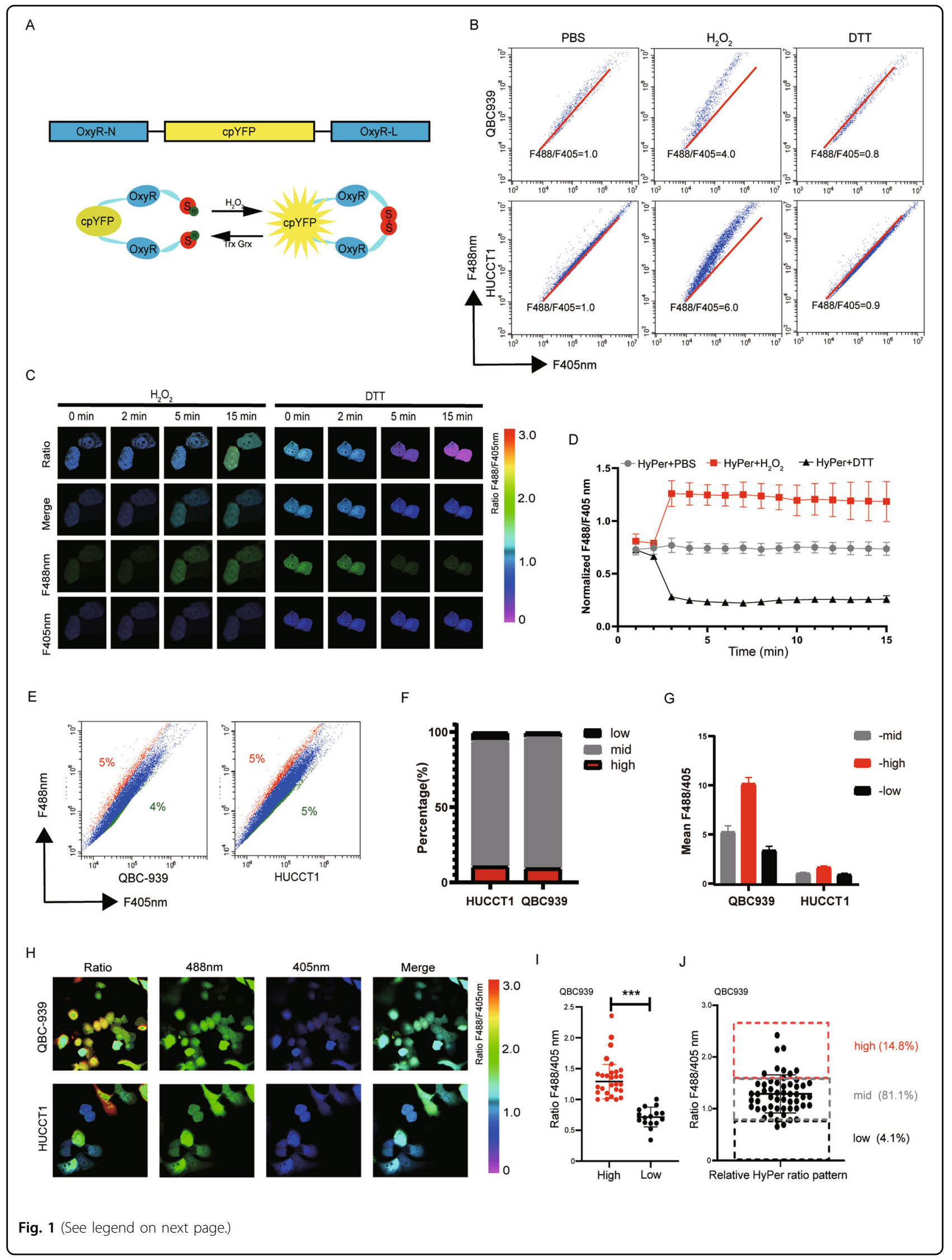


(see figure on previous page)

Fig. 1 The redox status was heterogeneous in cholangiocarcinoma, which was revealed by HyPer3. A The scheme of HyPer3 structure and its oxidation and reduction reaction. B, C QBC939 and HUCCT1 cells were transfected with HyPer3 plasmid for $48 \mathrm{~h}$ and the ratio of fluorescence (F488/ F405nm) was observed under flow-cytometric analysis and confocal microscopy with the treatment of PBS, hydrogen peroxide (100 $\mu \mathrm{M})$, and DTT $(5 \mathrm{mM})$, respectively. D Quantification of $\mathbf{C} . n=10$ cells/group. E Percentage of ROS differed subpopulations and their accurate ratio of QBC939 and HUCCT1 was measured by flow-cytometric analysis. F, G Quantification of E. Each experiment was performed in triplicate. $\mathbf{H}$ Percentage of ROS differed subpopulations and their accurate ratio of QBC939 and HUCCT1 was measured by confocal microscopy. I, J Quantification of $\mathbf{H}$. Each experiment was performed in triplicate. ${ }^{*} p<0.05 ;{ }^{* *} p<0.01 ;{ }^{* * *} p<0.001$.

fluorescence emission peak (F543 nm). Once under hydrogen peroxide stress, the intensity of the F405 nm excitation peak decreases, yet the F488 nm peak increases.

We first ectopically expressed HyPer3 in 2 kinds of human CCA cell lines, QBC939 and HUCCT1, and then determined the fluorescence ratio when excited at $488 \mathrm{~nm}$ and $405 \mathrm{~nm}$ when cells were in resting states or upon hydrogen peroxide or DTT treatment by flow cytometric analysis. Exogenous hydrogenous peroxide induced an instantaneous increase in the fluorescence ratios by $\sim 6.0$ fold in these two types of CCA cell lines, while exogenous DTT generally led to an inverse decrease in the fluorescence ratios by $\sim 0.8$-fold (Fig. 1B).

To further strictly confirm the sensitivity of the HyPer3 probe upon different stimulations, we subjected QBC939 cells to different treatments with hydrogen peroxide and DTT under confocal microscopy. The ratio of the HyPer3 probe fluorescence was markedly increased upon hydrogen peroxide stimulation and slightly decreased when NAC was added (Fig. 1C, D). Above all, we confirmed that the HyPer3 probe, which acts as a genetically encoded hydrogen peroxide biosensor, can dynamically reflect cellular ROS levels and the redox status under flow cytometric analysis or confocal microscopy.

We then examined the ROS level and redox status in the typical CCA cell lines QBC939 and HUCCT1. Following transfection of the HyPer3 probe into QBC939 and HUCCT1 cells, flow cytometric analysis and confocal microscopy tests were performed. Through flow cytometric analysis, we found that CCA cells exhibited a heterogeneous redox status, which was distinguished by the ratio of fluorescence of the HyPer3 probe, and we marked them as HyPer-mid, HyPer-high, and HyPer-low (Fig. 1E). Moreover, the proportions of each subgroup were close (Fig. 1F), while the ratio of the fluorescence of HyPer3 possessed a huge gap in that QBC939 tended to be more characterized among each subgroup (Fig. 1G). The confocal microscopy test showed similar results to the flow cytometric analysis in that the redox status of CCA cells was heterogeneous (Fig. 1H). Due to the larger gap from QBC939 to HUCCT1, we statistically calculated the ratio in QBC939 cells under confocal microscopy, which could be shown as a histogram with three distinct populations, HyPer-mid (81.1\% with a mean F488 nm/ F405 $\mathrm{nm}$ of $0.8 \sim 1.7)$, HyPer-high (14.8\% with a mean
F488 nm/F405 nm of 0.8 2.8) and HyPer-low (4.1\% with a mean F488 nm/F405 nm of 0 0.8) (Fig. 1I, J).

Above all, we found that the redox status of cholangiocarcinoma was heterogeneous and consisted of three distinct populations: HyPer-mid, HyPer-high, and HyPer-low. The subpopulations with different redox statuses can be efficiently sensitively distinguished by the genetically encoded biosensor HyPer3 probe.

\section{Different subpopulations of the cellular redox status in QBC939 elicited diverse chemoresistance to gemcitabine}

Having characterized that CCA cells were heterogeneous in their redox status, we aimed to identify the relationship between the cellular redox status and CCA gemcitabine chemoresistance by sorting the subpopulations with fluorescence-activated cell sorting (FACS) under the guidance of the HyPer3 probe. To control the sorting specificity, we checked the ratio of HyPer3 fluorescence in HyPer-high and HyPer-low cells (Fig. 2A). The sorted populations of QBC939 preserved the ratio gap between the HyPer-high and HyPer-low subpopulations (Fig. 2B). Furthermore we applied DCFHDA to make a further confirmation (Fig. 2C). The correlation was confirmed in the three subpopulations of QBC939, HyPer-mid, HyPer-high, and HyPer-low, by cellcounting kit 8 assays with gradient concentrations (from 0 to $100 \mu \mathrm{M}$ ) of gemcitabine, and HyPer-low CCA cells were characterized by higher resistance to gemcitabine (Fig. 2D). In a second complementary research approach, we used an apoptotic assay to identify the relationship between the redox status and gemcitabine chemoresistance (Fig. 2E, F). By Annexin-V/PI staining for flow cytometric analysis, the HyPer-low subpopulation of CCA cells exhibited a lower apoptosis rate than the HyPer-high subpopulation under gemcitabine treatment from 0 to $10 \mu \mathrm{M}$ (Fig. 2E, F). Moreover, the apoptotic assay using Hoechst immunofluorescence staining led the same conclusion as the apoptotic assay using Annexin-V/PI staining for flow cytometric analysis, in which the HyPer-low subpopulation of CCA cells possesses a higher rate of gemcitabine chemoresistance (Fig. 2G, H). Taken together, these data indicate that the great chemoresistance of the HyPer-low subpopulation to gemcitabine may be the inner reason for the chemoresistance of CCA to gemcitabine. 


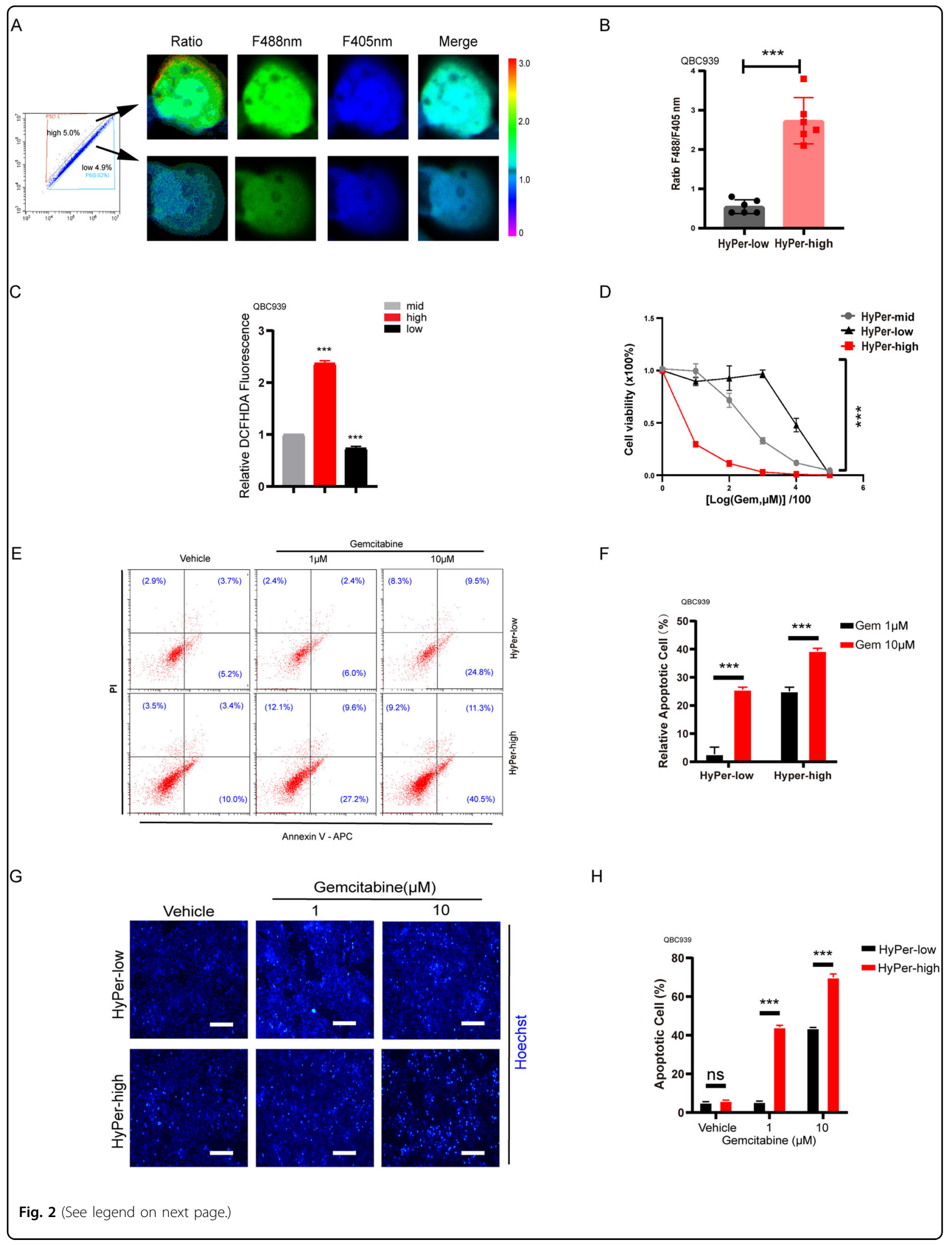


(see figure on previous page)

Fig. 2 Different subpopulations of the cellular redox status in QBC939 elicited diverse chemoresistance to gemcitabine. A QBC939 cells were transfected with the HyPer3 probe plasmid for $48 \mathrm{~h}$ and then were sorted by fluorescence-activated cell sorting. Sorted cells were checked by confocal microscopy. B Quantification of A. C Sorted cells were checked by DCFHDA under microplate reader. D Sorted cells were treated with Gemcitabine at the indicated concentrations for $48 \mathrm{~h}$. Cell viability was measured using CCK8. E The sorted cells were seeding in 6 well plates for $24 \mathrm{~h}$ and followed with gemcitabine treatment for $48 \mathrm{~h}$ at 1 and $10 \mu \mathrm{M}$. Apoptotic assay by Annexin-V/PI staining for flow cytometry after gemcitabine treatment for $48 \mathrm{~h}$. F Quantification of E. G Apoptotic assay by Hoechst dye for immunofluorescence after gemcitabine treatment for $48 \mathrm{~h}$. H Quantification of $\mathbf{G}$. Each experiment was performed in triplicate. ${ }^{*} p<0.05 ;{ }^{* *} p<0.01 ;{ }^{* * *} p<0.001$.

The different subpopulations of cellular redox status in QBC939 elicited diverse chemoresistance to gemcitabine in the xenograft nude mouse model

We further established that HyPer-low CCA cells possess higher gemcitabine chemoresistance than other subpopulations in vivo. We used a nude mouse model in which nude mice bearing tumors derived from HyPerhigh and HyPer-low CCA cells from QBC939 cells were treated with vehicle (PBS) and gemcitabine $(50 \mathrm{mg} / \mathrm{kg}$ body weight, intraperitoneal injection) every 2 days, and tumor volumes were measured at the same time (Fig. 3A). When HyPer-high and HyPer-low xenograft nude mice were treated with vehicle or gemcitabine, the HyPer-low subpopulation's tumor growth was not impaired by the gemcitabine compound but arrested when the Hyper-high subpopulation's tumor was treated with gemcitabine (Fig. 3B-D). Moreover, we further confirmed the differences in apoptosis and proliferation by immunofluorescence TUNEL and Ki-67 staining, which showed that the HyPer-low subpopulation possessed a lower apoptotic rate than the HyPer-high subpopulation under gemcitabine treatment, while a moderate decrease in proliferation was shared with no difference (Fig. 3E, F). Together, these data are consistent with the correlation between the different cellular redox statuses of CCA cells and gemcitabine chemoresistance, which is extremely severe in the HyPer-low subpopulation of CCA cells.

\section{MTHFD1 contributes to different cellular redox statuses and therefore chemoresistance to gemcitabine in cholangiocarcinoma}

Based on the higher gemcitabine chemoresistance in the HyPer-low subpopulation, we speculated that a key gene that encodes a reductase or an ROS scavenger may function. To delineate the relationship between cellular redox status and gemcitabine chemoresistance, we screened all of the candidates involved in reducing metabolism by quantitative real-time PCR. Among them, MTHFD1 was found to have the greatest increase in mRNA in HyPer-low CCA cells compared with HyPerhigh CCA cells (Fig. 4A). The data were replicated with a single-gene test by quantitative real-time PCR (Fig. 4B). Moreover, in addition to qPCR, western blot analysis further proved that HyPer-low CCA cells were characterized by higher MTHFD1 levels (Fig. 4D). To ensure cell line independence, we confirmed that the HyPer-low subpopulation of CCA cells possesses a higher level of MTHFD1 both in mRNA and protein in HUCCT1 (Fig. 4A, C, D). The evidence of relatively higher MTHFD1 expression in the HyPer-low subpopulation CCA cells than in the HyPer-high cells combined with the higher gemcitabine chemoresistance in the HyPer-low CCA cells encouraged us to determine whether MTHFD1 serves as one of the key regulators linking the cellular redox status with gemcitabine chemoresistance. MTHFD1 is one of the members of $\mathrm{C}-1$ tetrahydrofolate synthases that catalyses the interconversion of tetrahydrofolate (THF), 10-formyl-tetrahydrofolate, 5,10-methyltetrahydrofolate, and 5,10methylenetetrahydrofolate with NADPH generated as a by-product ${ }^{26}$. Due to that NADPH was generated as a byproduct followed with increased MTHFD1 expression, cellular $\mathrm{NADPH} / \mathrm{NADP}^{+}$ratio was detected in each subpopulation, respectively. At the basal level, HyPerlow CCA cells exhibited a higher $\mathrm{NADPH} / \mathrm{NADP}^{+}$ratio while HyPer-high cells go the reverse way which means the increased expression of MTHFD1 in HyPer-low cells will finally contribute to cellular NADPH increasing (Fig. $4 \mathrm{E})$. To determine the functions of MTHFD1 in cellular redox regulation and CCA gemcitabine chemoresistance, we silenced MTHFD1 in the human CCA cell line QBC939 and its HyPer-low subpopulation cells with a short interfering RNA (siRNA)-mediated gene knockdown approach (Fig. 4F), and siMTHFD1\#1 (siM1\#1) had a marked decrease in the mRNA levels of both QBC939 and its HyPer-low subpopulation cells (Fig. 4G). Meanwhile, with MTHFD1 knockdown, the ratio of cellular NADPH in HyPer-low cells experienced a dramatic decrease (Fig. 4H). Following MTHFD1 knockdown, both HyPer3 QBC939 cells and HyPer-low subpopulation cells exhibited a notable increase in the fluorescence ratio, while the antioxidant and free radical scavenger $\mathrm{N}$-acetyl-L-cysteine (NAC) effectively reversed the fluorescence ratio increase (Fig. 4I, J). We next asked whether knockdown of MTHFD1 altered the gemcitabine chemoresistance in the HyPer-low subpopulation CCA cells. Compared with the HyPer-high subpopulation CCA cells, CCK8 assays indicated that HyPer-low CCA cells with 


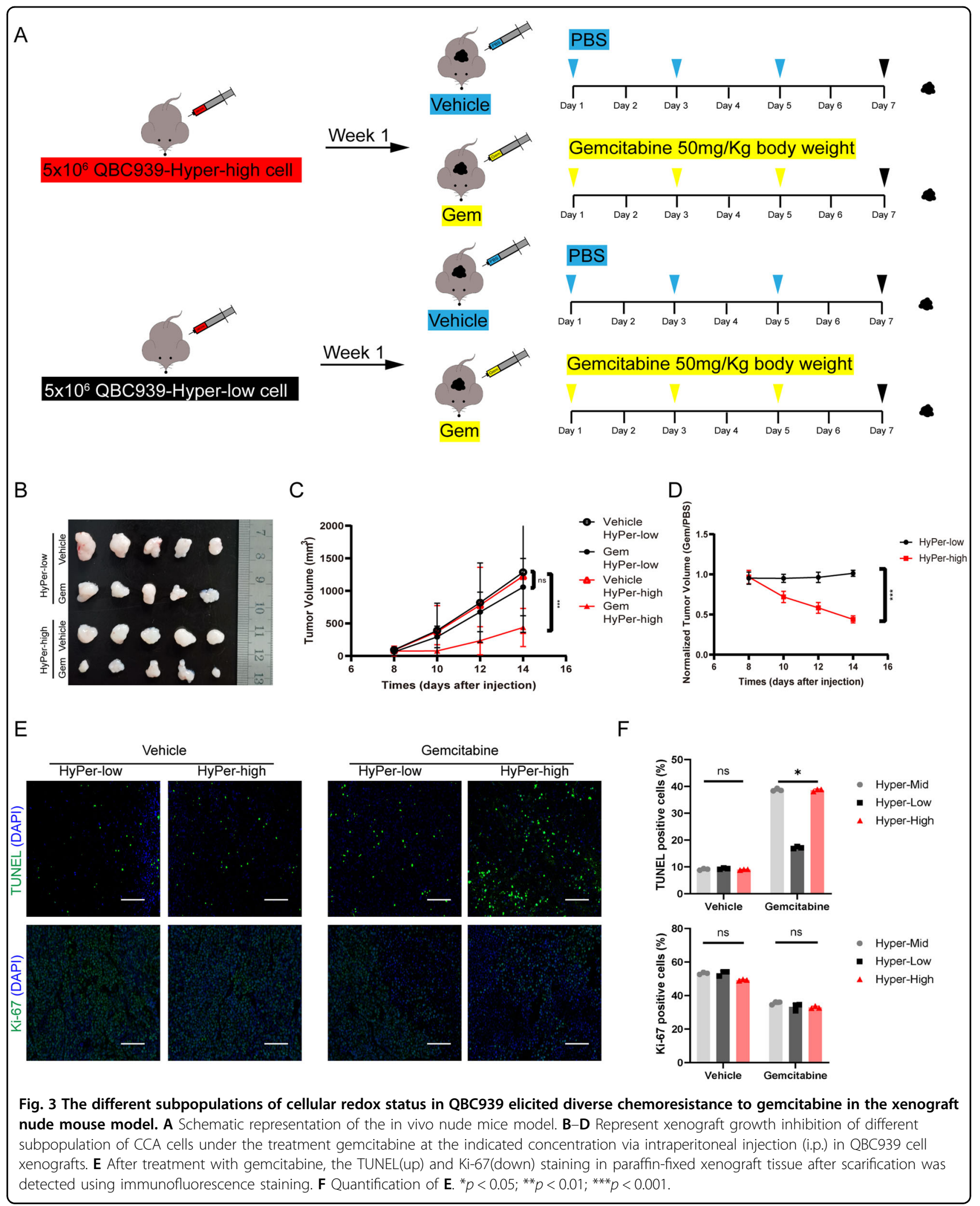




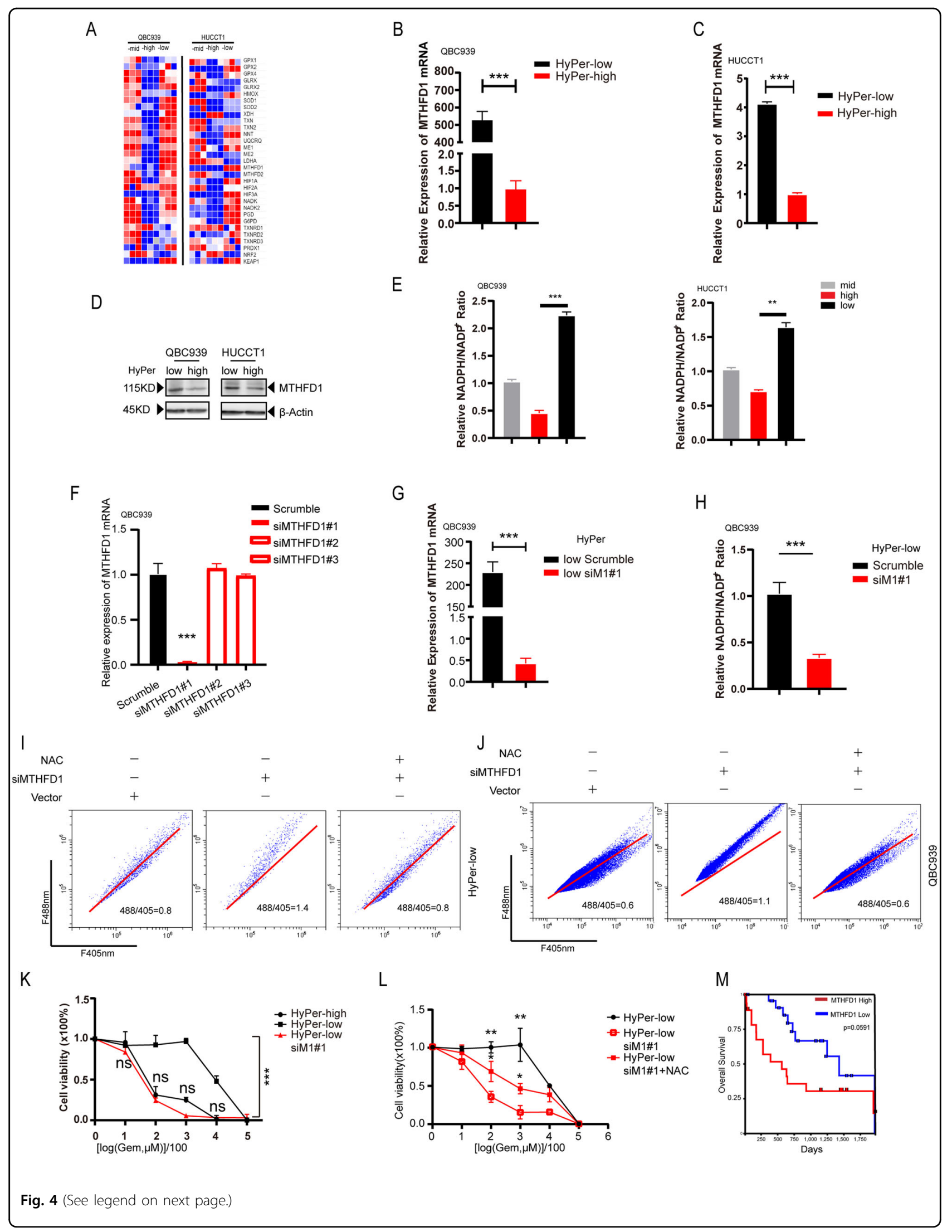


(see figure on previous page)

Fig. 4 MTHFD1 contributes to different cellular redox statuses and therefore chemoresistance to gemcitabine in cholangiocarcinoma. A Heat map of reductase encoded gene in each subpopulation by quantitative real-time PCR. B, C Relative expression of MTHFD1 mRNA in QBC939 and HUCCT1 respectively by quantitative real-time PCR. D HyPer-Low subpopulation of CCA cells possess a higher expression level of MTHFD1 protein. E Ratio of cellular NADPH/NADP ${ }^{+}$in each subpopulation by microplate reader. F, G MTHFD1 knockdown efficiency by short interfering RNA was detected by quantitative real-time PCR. $\mathbf{H}$ Ratio of cellular NADPH/NADP ${ }^{+}$in Scumble-treat and siM1\#1 treat HyPer-low CCA cells by microplate reader. I, J QBC939 and HyPer-Low subpopulation of CCA cells were treated with short interfering RNA of MTHFD1, or its vector or NAC. The ratio of fluorescence was detected by flow-cytometric analysis. K, L HyPer-High and HyPer-Low subpopulation of CCA cells and siM1\#1 HyPer-Low cells were treated with Gemcitabine or NAC at the indicated concentrations for $48 \mathrm{~h}$. Cell viability was measured using CCK8. $\mathbf{M}$ Quantification of overall survival data from TCGA database. Each experiment was performed in triplicate. ${ }^{*} p<0.05 ;{ }^{* *} p<0.01 ;{ }^{* * *} p<0.001$.

higher expression levels of MTHFD1 exhibited significant chemoresistance to gemcitabine, whereas MTHFD1 knockdown transformed the gemcitabine chemoresistance of HyPer-low CCA cells into sensitivity, and this transformation could be reversed by NAC treatment (Fig. 4K, L). With further confirmation, the overall survival rate data from the TCGA database displayed a difference between cholangiocarcinoma patients with higher or lower expression of MTHFD1 (Fig. 4M). Taken together, we proved that MTHFD1 overexpression links the cellular redox status with enhanced gemcitabine chemoresistance in the HyPer-low subpopulation CCA cells and cholangiocarcinoma cells.

\section{Methotrexate synergized with gemcitabine in cholangiocarcinoma via MTHFD1-mediated ROS restoration}

Based on the transformation from gemcitabine chemoresistance to sensitivity after MTHFD1 was knocked down, we hypothesized that antifolates might synergize with gemcitabine to cure patients who are resistant to gemcitabine. Methotrexate (MTX), an antifolate compound, was considered a candidate with great possibility due to its MTHFD1 inhibiting effect on dihydrofolate reductase (DHFR) and reduced cellular NADPH at the same time. To test this hypothesis, we applied MTX and gemcitabine alone and in combination with QBC939 and its HyPer-low subpopulation CCA cells. Alone with MTX treatment, MTHFD1 mRNA and its protein exhibited a great decrease in both QBC939 and its HyPer-low subpopulation CCA cells (Fig. 5A-C). We next tested whether the cellular redox status of QBC939 was affected by MTX treatment. Compared with the control treatment of PBS, the fluorescence ratio of the HyPer3 probe by flow cytometric analysis revealed that MTX treatment significantly increased cellular ROS (Fig. 5D). Following the increased cellular ROS, the apoptosis rate was increased at the same time, while the difference in apoptosis between HyPer-low and HyPer-high CCA cells also disappeared by Hoechst immunofluorescence staining (Fig. 5E). For further confirmation, a dose-dependent manner of MTX combine with gemcitabine was adopt. In contrast to the poor response to gemcitabine individually in the HyPerlow subpopulation of CCA cells, the CCK-8 assay proved that the combination of MTX and gemcitabine impaired cell viability in HyPer-low CCA cells under gemcitabine treatment (Fig. 5F) and Annexin-V/PI staining for flow cytometric analysis reassure our conclusion (Fig. 5G, H).

\section{Discussion}

In the present study, working with a novel geneencoded fluorescence probe that is sensitive to hydrogen peroxide, we sorted the cellular redox statuses of different subpopulations of CCA cells and identified a determinate subpopulation of CCA cells and genes that are essential for gemcitabine chemoresistance in CCA. Our data show that the HyPer-low subpopulation, which is characterized by a lower cellular redox status, is highly associated with gemcitabine chemoresistance. The key link between lower cellular redox status and gemcitabine chemoresistance is the high expression of MTHFD1 in the HyPer-low subpopulation of CCA cells. Mechanistically, as a key enzyme that catalyses the transversion of tetrahydrofolate (THF), 10-formyl-tetrahydrofolate, 5,10-methyltetrahydrofolate, and 5,10-methylenetetrahydrofolate ${ }^{26}$, NADPH was generated as a by-product from the reduction of NADP, and the catalytic reaction and cellular redox status were changed. As a result of increased cellular NAPDH, gemcitabine-induced apoptosis was efficiently inhibited, which could be restored by MTHFD1 knockdown. Based on the above research, we provide a potential treatment strategy in which a combination of gemcitabine and the antifolate compound MTX can effectively impair tumor growth in cholangiocarcinoma resulting from restored cellular ROS content. We support the hypothesis that MTHFD1 overexpression related to a lower cellular redox status leads to gemcitabine chemoresistance in CCA cells, which is highly associated with the much lower cell viability of HyPer-high than HyPer-low CCA cells under gemcitabine treatment, indicating that MTHFD1 expression is highly correlated with gemcitabine chemoresistance in CCA cells (Fig. 6).

As an antitumour chemotherapeutic due to its role as a cytidine analog, gemcitabine efficiently works with its metabolites dFdCDP and dFdCTP, which can decrease competing deoxyribonucleotide pools and lead to the termination of DNA chain elongation, respectively ${ }^{27}$. In 


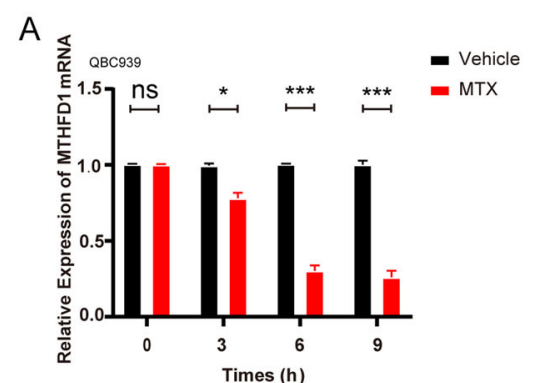

D

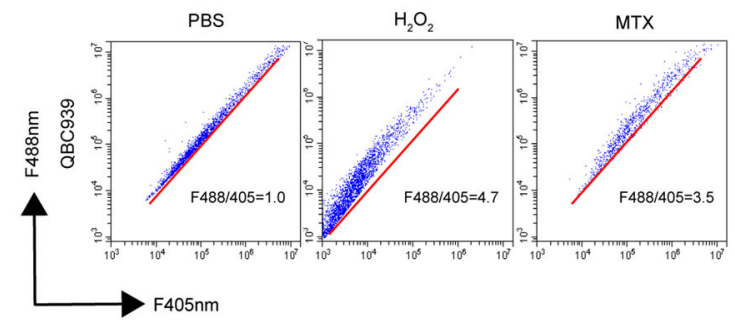

$\mathrm{F}$

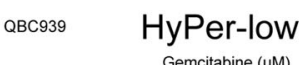

Gemcitabine $(\mu \mathrm{M})$ \begin{tabular}{llllllll}
0 & 0.1 & 1 & 3 & 5 & 7 & 9 & 10 \\
\hline
\end{tabular}

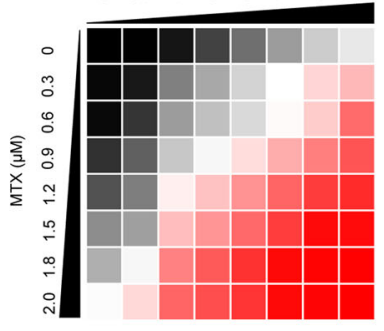

G
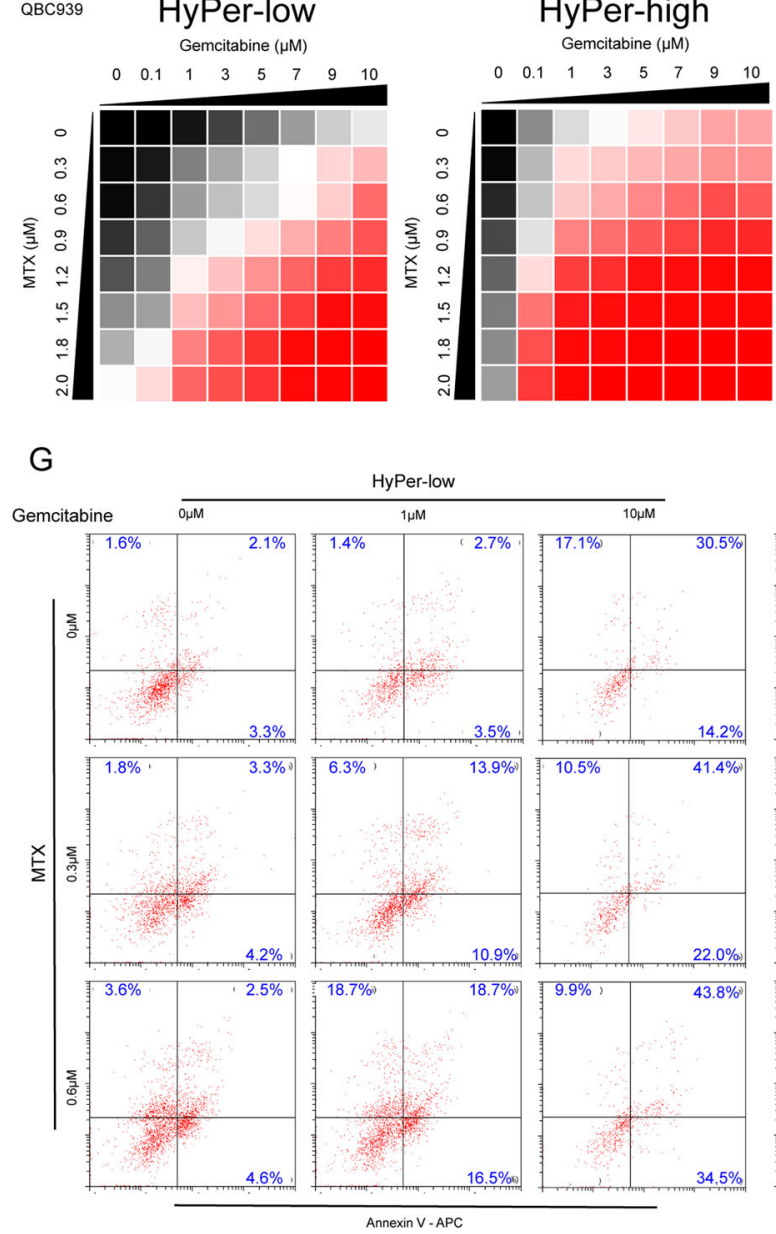

B

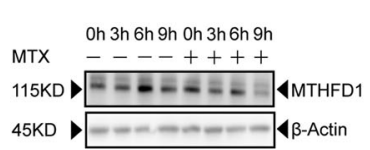

$\mathrm{C} \underset{\frac{\pi}{\varepsilon}}{\stackrel{\pi}{\pi}}$

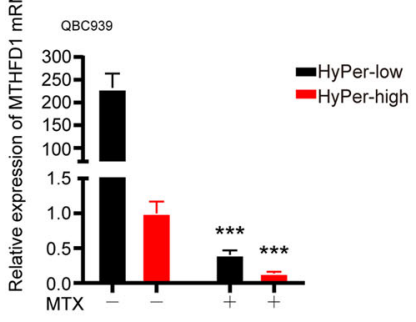

E

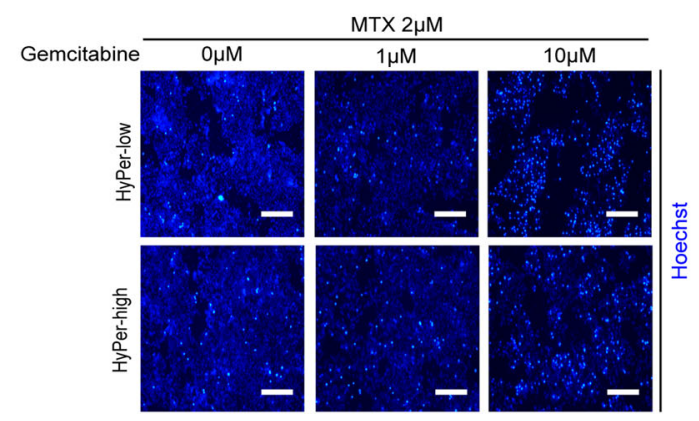

$\mathrm{H}$
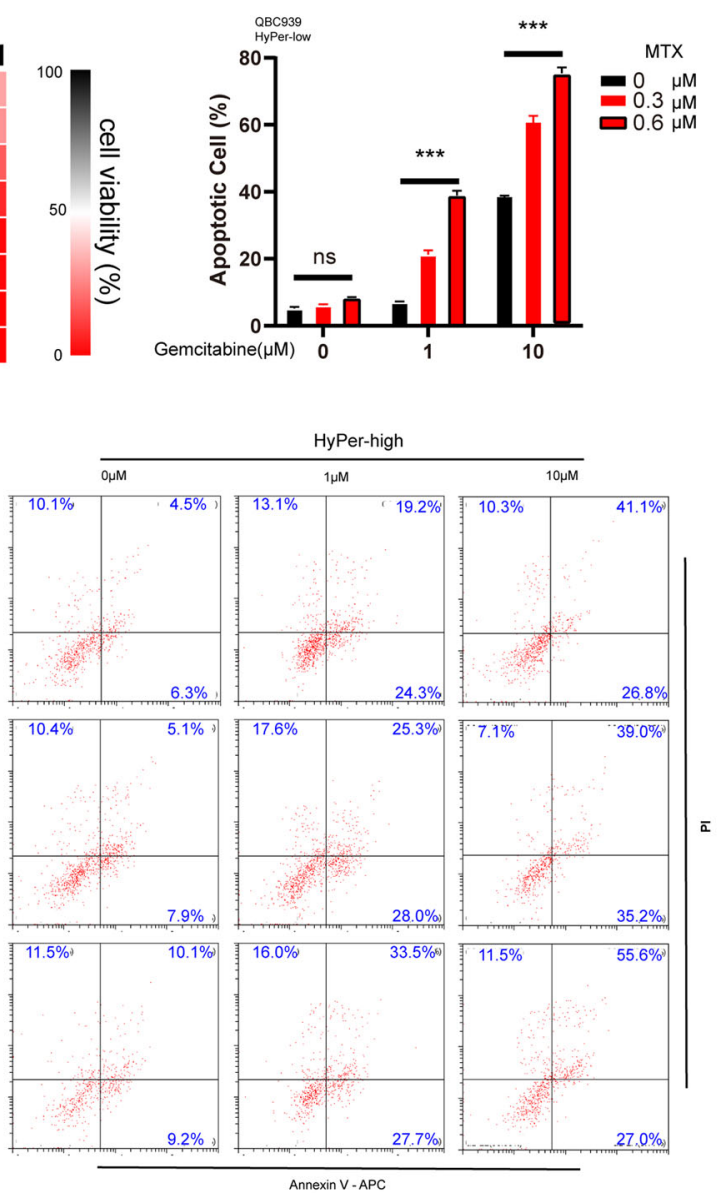

Fig. 5 (See legend on next page.) 
(see figure on previous page)

Fig. 5 Methotrexate synergized with gemcitabine in cholangiocarcinoma via MTHFD1-mediated ROS restoration. A, B QBC939 was treated with MTX at the indicated concentrations or PBS for $48 \mathrm{~h}$. Relative expression of MTHFD1 of mRNA and protein was measured. C HyPer-Low and HyPer-High subpopulations were treated with MTX at the indicated concentrations for $48 \mathrm{~h}$. Relative expression of MTHFD1 mRNA was measured. D QBC939 was treated with PBS, $\mathrm{H}_{2} \mathrm{O}_{2}$, and MTX respectively at the indicated concentration. The ratio of the HyPer3 probe was measured by flowcytometric analysis. E The sorted cells were seeded in 6 well plates for $24 \mathrm{~h}$ and followed with gemcitabine treatment for $48 \mathrm{~h}$ at 1 and $10 \mu \mathrm{M}$ combine with MTX. Apoptotic assay by Hoechst dye for immunofluorescence after gemcitabine treatment for $48 \mathrm{~h}$. $\mathbf{F}$ Sorted cells were treated with Gemcitabine at the indicated concentrations with MTX at indicated concentrations respectively for $48 \mathrm{~h}$. Cell viability was measured using CCK8. G Apoptotic assay by Annexin-V/PI staining for flow cytometry after treated with gemcitabine at the indicated concentrations with MTX at indicated concentrations respectively for $48 \mathrm{~h}$ for sorted cells. $\mathbf{H}$ Quantification of $\mathbf{G}$. Each experiment was performed in triplicate. ${ }^{*} p<0.05 ;{ }^{* *} p<0.01$; ${ }^{* * *} p<0.001$.

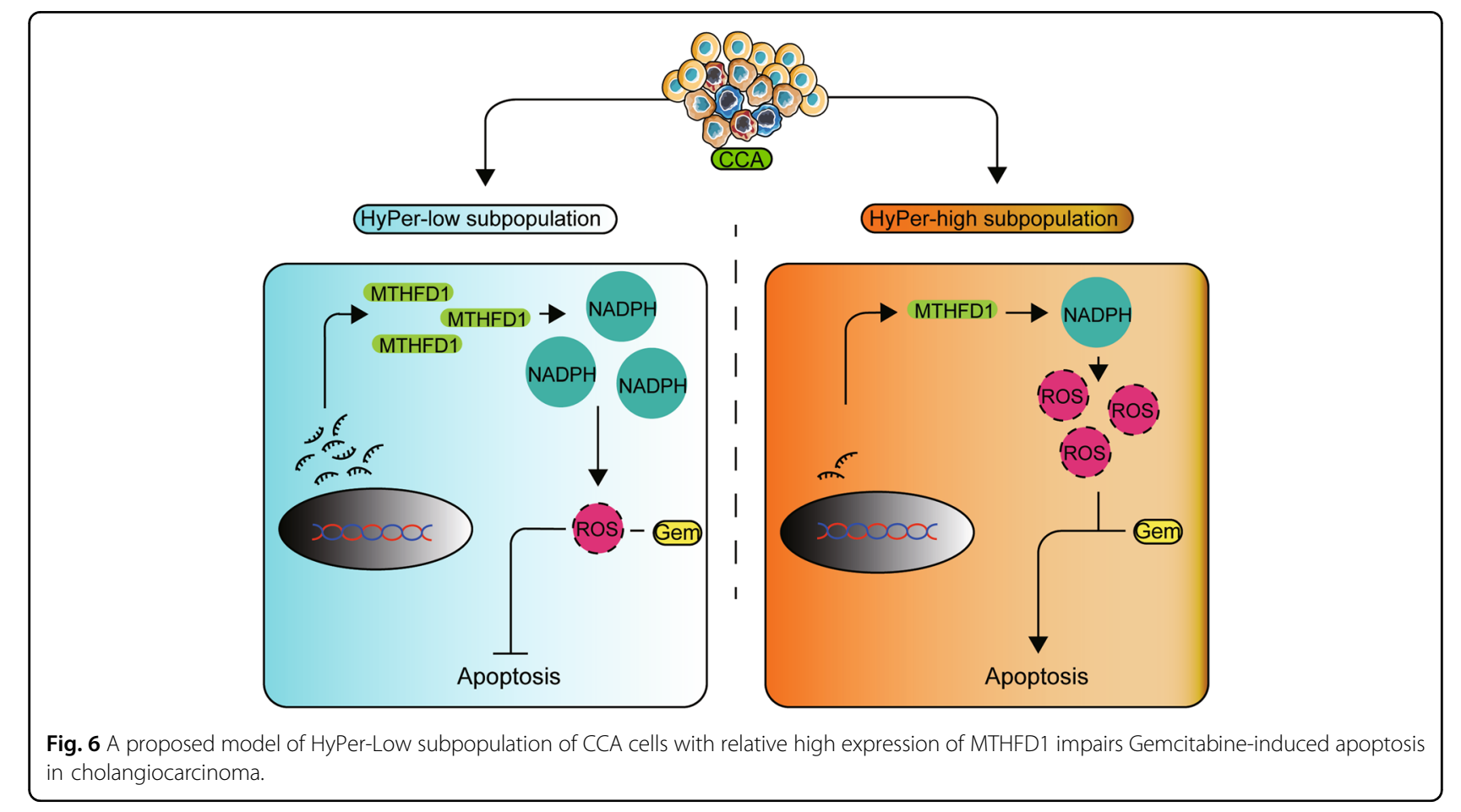

clinical practice over the years, gemcitabine has been broadly used for its capacity to induce apoptosis either single-use in pancreatic cancer or combined-use in nonsmall cell lung cancer, bladder cancer, breast cancer, etc., with unclear mechanisms ${ }^{28-32}$. In recent decades, an increasing number of studies have confirmed that the cellular redox status or reactive oxygen species (ROS) play an important role in gemcitabine-induced cancer cell apoptosis via direct killing or signal transduction ${ }^{33,34}$. We also confirmed that CCA cells with different cellular ROS exhibited distinct gemcitabine responses. As Huanchen Sha et al. and Yunfeng Zhao et al. reported, ROS-related genes such as NRF2 and $U C P 2$ can impair gemcitabineinduced apoptosis by downregulating NAF-1 or enhancing the stemness of cancer cells ${ }^{10,35}$. Unexpectedly but reasonably, we found that the severe gemcitabine chemoresistance in CCA, especially in the HyPer-low subpopulation of CCA cells, was due to the relatively high expression of MTHFD1, the key enzyme in folate metabolism. Under the catalysis of MTHFD1 during the transversion of the isoforms of THF, NADPH was produced as a by-product and imbalanced the cellular redox status. In the past, the role of MTHFD1 in folate metabolism was believed to thoroughly influence the recurrence of $\mathrm{HCC}^{21}$, neural tube defects ${ }^{36}$, and melanoma metastasis $^{37}$ and induce intestinal carcinogenesis ${ }^{38}$. In the past 5 years, MTHFD1 was found to regulate hypertension by DNA methylation ${ }^{39}$ and regulate transcription by interacting with $B R D 4^{26}$. Compared to the above studies, our findings proved that MTHFD1 is quite important for CCA's gemcitabine chemoresistance but further found that the correlation between higher expression of MTHFD1 and gemcitabine chemoresistance resulted in an influence on the cellular redox status due to the overproduction of NADHP under this condition. Once MTHFD1 was knocked down by short interfering RNA, 
severe gemcitabine chemoresistance was greatly alleviated, accompanied by the differences between the cellular redox statuses of the HyPer-low and HyPer-high subpopulations being effectively canceled. However, the results can also be complicated because of MTHFD1's enzyme activity. In addition, MTHFD1 also plays an important role in the stemness of cancer cells, while cancer stem cells are also highly associated with chemoresistance ${ }^{40}$. Our data effectively exhibit the severe gemcitabine chemoresistance in HyPer-low CCA cells with high expression of MTHFD1 but neglect to verify the potential possibility that HyPer-low CCA cells have equal CCA stem cells, which should be completed in our future study.

Due to the vigorous proliferation of tumor cells, pathways or processes involved were of concern, folate metabolism was greatly highlighted ${ }^{41-43}$ and antifolate compounds such as MTX were developed. Once again, MTX was considered to be effective by inhibiting cell division and nucleotide/deoxynucleotide synthesis ${ }^{44,45}$. In detail, MTX inhibits dihydrofolate reductase leading to depletion of tetrahydrofolate, which is involved in multiple chemical reactions of homocysteine to methionine, where it acts as a proximal methyl donor. Methionine then transformed into S-adenosylmethionine (SAM), which provides methyl groups for RNA, DNA et al. and finally decreased MTHFD1 expression. Our data suggest a new target for MTX, which could act as a new strategy to cure CCA patients with gemcitabine chemoresistance. Furthermore, the combination of MTX and gemcitabine is highly recommended as a new strategy for CCA patients who are resistant to gemcitabine.

\section{Acknowledgements}

The authors thank Yuzheng Zhao and Yi Yang for providing us with HyPer3 selflessly. This work was supported by the National Natural Science Foundation of China (81600491).

\section{Author details \\ 'Department of Biliary and Pancreatic Surgery, Renji Hospital, School of Medicine, Shanghai Jiaotong University, Shanghai 200127, China. ${ }^{2}$ Department of Biochemistry and Molecular Cell Biology, State Key Laboratory of Oncogenes and Related Genes, Shanghai Key Laboratory forTumor Microenvironment and Inflammation, Shanghai Jiaotong University School of Medicine, Shanghai 200025, China. ${ }^{3}$ Optogenetics \& Synthetic Biology Interdisciplinary Research Center, State Key Laboratory of Bioreactor Engineering, Shanghai Collaborative Innovation Center for Biomanufacturing Technology, East China University of Science and Technology, Shanghai, China. ${ }^{4}$ Shanghai Key Laboratory of New Drug Design, School of Pharmacy, East China University of Science and Technology, Shanghai, China. ${ }^{5}$ CAS Center for Excellence in Brain Science, Shanghai Institutes for Biological Sciences, Chinese Academy of Sciences, Shanghai, China}

\section{Author contributions}

R.P., K.L., J.Y., and X.S. designed and performed the experiments and wrote the manuscript. Z.Y., G.W., X.W., and J.Q. assisted with experiments. Y.Z., and Y.Y., donated the HyPer3 plasmid. K.L., J.Y., X.S., and Y.L., supervised and admitted this project.
Conflict of interest

The authors declare no competing interests.

\section{Ethics statement}

The animal study was reviewed and approved by the Ethics Committee of Renji Hospital.

\section{Publisher's note}

Springer Nature remains neutral with regard to jurisdictional claims in published maps and institutional affiliations.

Received: 31 March 2021 Accepted: 7 April 2021

Published online: 01 May 2021

\section{References}

1. Rizvi, S., Khan, S. A.r Hallemeier, C. L., Kelley, R. K. \& Gores, G. J. Cholangiocarcinoma - evolving concepts and therapeutic strategies. Nat. Rev. Clin. Oncol. 15, 95-111 (2018)

2. Razumilava, N. \& Gores, G. J. Cholangiocarcinoma. Lancet 383, 2168-2179 (2014).

3. Howlader N. N. A., et al. (eds). SEER Cancer Statistics Review. National Cancer Institute; 2019.

4. Jarnagin, W. R. et al. Staging, resectability, and outcome in 225 patients with hilar cholangiocarcinoma. Ann. Surg. 234, 507-517 (2001). discussion 517-509.

5. Valle, J. et al. Cisplatin plus gemcitabine versus gemcitabine for biliary tract cancer. N. Engl. J. Med. 362, 1273-1281 (2010).

6. Abdel-Rahman, O., Elsayed, Z. \& Elhalawani, H. Gemcitabine-based chemotherapy for advanced biliary tract carcinomas. Cochrane Database Syst. Rev. 4, CD011746 (2018).

7. Binenbaum, Y., Na'ara, S. \& Gil, Z. Gemcitabine resistance in pancreatic ductal adenocarcinoma. Drug Resist. Updat. 23, 55-68 (2015).

8. Yokoi, K. \& Fidler, I. J. Hypoxia increases resistance of human pancreatic cancer cells to apoptosis induced by gemcitabine. Clin. Cancer Res. 10, 2299-2306 (2004).

9. Meng, Q. et al. Abrogation of glutathione peroxidase-1 drives EMT and chemoresistance in pancreatic cancer by activating ROS-mediated Akt/GSK3beta/ Snail signaling. Oncogene 37, 5843-5857 (2018).

10. Yu J., Shi L., Lin W., Lu B., Zhao Y. UCP2 promotes proliferation and chemoresistance through regulating the NF-KB/ $\beta$-catenin axis and mitochondrial ROS in gallbladder cancer. Biochemical Pharmacol. 172 113745 (2020).

11. Wei, L. et al. Genome-wide CRISPR/Cas9 library screening identified PHGDH as a critical driver for Sorafenib resistance in HCC. Nat. Commun. 10, 4681 (2019).

12. $\mathrm{Du}, \mathrm{H}$. et al. Elevated glutathione peroxidase 2 expression promotes cisplatin resistance in lung adenocarcinoma. Oxid. Med. Cell Longev. 2020, 7370157 (2020).

13. Chen, G. et al. Different redox states in malignant and nonmalignant esophageal epithelial cells and differential cytotoxic responses to bile acid and honokiol. Antioxid. Redox Signal 11, 1083-1095 (2009).

14. Wang, S. et al. PRDX2 protects against oxidative stress induced by H. pylori and promotes resistance to cisplatin in gastric cancer. Redox Biol. 28, 101319 (2020).

15. Cui, Y., She, K., Tian, D., Zhang, P. \& Xin, X. miR-146a inhibits proliferation and enhances chemosensitivity in epithelial ovarian cancer via reduction of SOD2. Oncol. Res. 23, 275-282 (2016).

16. D'Autreaux, B. \& Toledano, M. B. ROS as signalling molecules: mechanisms that generate specificity in ROS homeostasis. Nat. Rev. Mol. Cell Biol. 8, 813-824 (2007).

17. Marengo, B. et al. Redox homeostasis and cellular antioxidant systems: crucial players in cancer growth and therapy. Oxid. Med. Cell Longev. 2016, 6235641 (2016).

18. Sosa, V. et al. Oxidative stress and cancer: an overview. Ageing Res. Rev. 12 376-390 (2013).

19. Trachootham, D., Alexandre, J. \& Huang, P. Targeting cancer cells by ROSmediated mechanisms: a radical therapeutic approach? Nat. Rev. Drug Disco. 8, 579-591 (2009). 
20. Yin, X. et al. ID1 promotes hepatocellular carcinoma proliferation and confers chemoresistance to oxaliplatin by activating pentose phosphate pathway. J. Exp. Clin. Cancer Res. 36, 166 (2017).

21. $\mathrm{Yu}, \mathrm{H}$. et al. Overexpression of MTHFD1 in hepatocellular carcinoma predicts poorer survival and recurrence. Future Oncol. 15, 1771-1780 (2019).

22. Bilan, D. S. et al. HyPer-3: a genetically encoded $\mathrm{H}_{(2)} \mathrm{O}_{(2)}$ probe with improved performance for ratiometric and fluorescence lifetime imaging. ACS Chem. Biol. 8, 535-542 (2013).

23. Belousov, V. V. et al. Genetically encoded fluorescent indicator for intracellular hydrogen peroxide. Nat. Methods 3, 281-286 (2006).

24. Bilan, D. S. \& Belousov, V. V. HyPer family probes: state of the art. Antioxid. Redox Signal 24, 731-751 (2016).

25. Bilan, D. S. \& Belousov, V. V. In vivo imaging of hydrogen peroxide with HyPer probes. Antioxid. Redox Signal. 29, 569-584 (2018).

26. Sdelci, S. et al. MTHFD1 interaction with BRD4 links folate metabolism to transcriptional regulation. Nat. Genet 51, 990-998 (2019).

27. Mini, E., Nobili, S., Caciagli, B., Landini, I. \& Mazzei, T. Cellular pharmacology of gemcitabine. Ann. Oncol. 17, v7-v12 (2006). Suppl 5.

28. Burris, H. A. et al. Improvements in survival and clinical benefit with gemcitabine as first-line therapy for patients with advanced pancreas cancer: a randomized trial. J. Clin. Oncol. 15, 2403-2413 (1997).

29. Mornex, F. \& Girard, N. Gemcitabine and radiation therapy in non-small cell lung cancer: state of the art. Ann. Oncol. 17, 1743-1747 (2006).

30. Monnerat, C., Le \& Chevalier, T. Review of the pemetrexed and gemcitabine combination in patients with advanced-stage non-small cell lung cancer. Ann. Oncol. 17, v86-v90 (2006). Suppl 5.

31. Coen, J. J. et al. Bladder preservation with twice-a-day radiation plus fluorouracil/cisplatin or once daily radiation plus gemcitabine for muscle-invasive bladder cancer: NRG/RTOG 0712A randomized phase II trial. J. Clin. Oncol. 37, 44 (2019)

32. Hu, X.-C. et al. Cisplatin plus gemcitabine versus paclitaxel plus gemcitabine as first-line therapy for metastatic triple-negative breast cancer (CBCSG006): a randomised, open-label, multicentre, phase 3 trial. Lancet Oncol. 16, 436-446 (2015).
33. Patel, G. K. et al. Exosomes confer chemoresistance to pancreatic cancer cells by promoting ROS detoxification and miR-155-mediated suppression of key gemcitabine-metabolising enzyme, DCK. Br. J. Cancer 116, 609-619 (2017).

34. Ahmmed, B. et al. Rg3 inhibits gemcitabine-induced lung cancer cell invasiveness through ROS-dependent, NF-KB- and HIF-1a-mediated downregulation of PTX3. J. Cell. Physiol. 234, 10680-10697 (2019).

35. Cheng, L. et al. Resveratrol-induced downregulation of NAF-1 Enhances the Sensitivity of Pancreatic Cancer Cells to Gemcitabine via the ROS/Nrf2 Signaling Pathways. Oxid. Med Cell Longev. 2018, 9482018 (2018).

36. Meng, J., Han, L. \& Zhuang, B. Association between MTHFD1 polymorphisms and neural tube defect susceptibility. J. Neurol. Sci. 348, 188-194 (2015).

37. Piskounova, E. et al. Oxidative stress inhibits distant metastasis by human melanoma cells. Nature 527, 186-191 (2015).

38. MacFarlane, A. J., Perry, C. A., McEntee, M. F., Lin, D. M. \& Stover, P. J. Mthfd1 is a modifier of chemically induced intestinal carcinogenesis. Carcinogenesis $\mathbf{3 2}$ 427-433 (2011).

39. $\mathrm{Xu}, \mathrm{M}$. et al. MTHFD1 promoter hypermethylation increases the risk of hypertension. Clin. Exp. Hypertens. 41, 422-427 (2019).

40. Stumpf, A. N. et al. Identification of 4 new HLA-DR-restricted minor histocompatibility antigens as hematopoietic targets in antitumor immunity. Blood 114, 3684-3692 (2009).

41. Pieroth, R., Paver, S., Day, S. \& Lammersfeld, C. Folate and its impact on cancer risk. Curr. Nutr. Rep. 7, 70-84 (2018).

42. Moazzen, S. et al. Folic acid intake and folate status and colorectal cancer risk: a systematic review and meta-analysis. Clin. Nutr. 37, 1926-1934 (2018). (6 Pt A).

43. Crider, K. S., Yang, T. P., Berry, R. J. \& Bailey, L. B. Folate and DNA methylation: a review of molecular mechanisms and the evidence for folate's role. Adv. Nutr. 3, 21-38 (2012)

44. Pope, J. E. et al. Treatment patterns in rheumatoid arthritis after discontinuation of methotrexate: data from the Ontario Best Practices Research Initiative (OBRI). Clin. Exp. Rheumatol. 36, 215-222 (2018).

45. Cronstein, B. N. The mechanism of action of methotrexate. Rheum. Dis. Clin. N. Am. 23, 739 (1997). 